\title{
IDENTIFICATION OF STABLE GENOTYPE THROUGH STABILITY ANALYSIS IN WHEAT (Triticum aestivum L.)
}

\author{
M.D. Rajput, P. N. Rasal, V. S. Girase and D. A. Gadekar \\ Botany Section, College of Agriculture, Dhule, (Maharashtra) India \\ E-Mail: vikramgirase@gmail.com
}

\begin{abstract}
:
The present investigation entitled "Adaptability Parameters in Wheat" was carried out to study the nature and performance of wheat genotypes at various environments for the yield and yield attributing characters. Twelve genotypes and four released varieties of wheat were grown at Botany Section Farm, College of Agriculture, Dhule during Rabi 2014-15, in a Randomized Block Design with three replications across four environments.

The genotypes NIAW 2565, NIAW 2563, NIAW 34 and NIAW 2556 were found promising in E3 and E4 environments, hence these genotypes can be considered in breeding programmes for improvement of wheat for late sowing. The values of $\mathrm{G} \times \mathrm{E}$ interaction were significant for all the twelve characters, which indicated that genotypes interacted differently with environmental variations. With respect to grain yield, NIAW 2495, NIAW 2595, NIAW 2539 and NIAW 301 were found to have below average stability and are suitable for favorable environment. Whereas, NIAW 1994 was grouped in above average stable and is suitable for unfavorable environment. Based on per se performance, regression coefficient and deviations from regression, the genotypes viz; NIAW 2565, NIAW 2556 and NIAW 2563 were found to be stable and adaptable to a wide range of environments suggesting that these genotypes be better exploited in terms of grain yield.
\end{abstract}

Key words:- Adaptability, Stability, GXE interactions, Wheat

\section{INTRODUCTION:}

Wheat belongs to family Poaceae (Gramineae). The chromosome number sets (genomes) for wheat are diploids $14(\mathrm{n}=7)$, tetraploids $28(n=14)$ and hexaploids $42(n=21)$. There are 50 wild species of wheat, out of which four species viz. Triticum aestivum, Triticum durum, Triticum diccocum and Triticum sphaerococcum are under cultivation in India. Triticum aestivum is the most important species occupying more than 90 per cent of the total wheat area in country, followed by Triticum durum (8-9 \%) and Triticum diccocum (< 1\%). Triticum sphaerococcumis not under cultivation due to its susceptibility to rust diseases and poor yields. Any breeding programme is aimed at development of genotypes having high yielding, stable, resistance or tolerance to biotic and abiotic stresses with good quality attributes, showing good response and stability under different environmental conditions. Information on impact of sowing dates is thus very important to adjust the genotypes for their stability in wide range of environments. The $G \quad x \quad E$ interactions may offer opportunities especially in the selection and adoption of genotypes, showed positive interaction with location and its prevailing environmental conditions (exploitation of yield stability). Improvement of end use quality in bread wheat depends on thorough understanding of current wheat quality and the influence of genotypes, environments and genotype $\mathrm{x}$ environment interactions on quality traits. Moreover, stability of quality characters is also important in increasing selection efficiency for breeders 
in plant breeding programme. For farmers point of view, stability analysis it is very essential to assess the suitability of variety or genotype for early, timely and late sowing.

\section{MATERIAL \& METHODS:}

The experimental materials consisted of twelve new bread wheat genotypes and four released varieties developed at Agricultural Research Station, Niphad, were used for assessing the stability of different genotypes at multienvironments for grain yield, its components and quality traits. The material were grown in four different environment on 31.10.2014 (E1), 16.11.2014 (E2), 1.12.2014) (E3) and 16.12.2014(E4). The observations were recorded on the 12 characters viz; days to $50 \%$ flowering, days to maturity, plant height $(\mathrm{cm})$, number of tillers meter-1, earhead length, number of spikelets earhead-1, number of grains earhead-1, 1000-grain weight $(\mathrm{g})$, grain yield plot $^{-1}(\mathrm{~g})$, protein content $(\%)$, sedimentation value $(\mathrm{ml})$ and hectolitre weight $(\mathrm{kg} / \mathrm{hl})$. Phenotypic stability of a genotypes for these characters was estimated by regression analysis as per the procedure Eberhart and Russell (1966). According to this model, a desirable variety with good stability should have high mean $(\mu)$, unit regression coefficient $(b i=1)$ and the minimum deviation i.e. equal to zero or non-significant from regression $\left(\mathrm{S}^{2} \mathrm{di}=0\right)$.

\section{RESULT AND DISCUSSION:}

The present investigation entitled "Adaptability Parameters in Wheat" was carried out to study the nature and performance of wheat genotypes at various environments for the different yield attributing characters. Twelve genotypes and four released varieties of wheat were grown at Botany Section Farm, College of Agriculture, Dhule during Rabi 2014-15, in a Randomized Block Design with three replications across the four environments.
Each genotype was grown in four rows spaced at $22.5 \mathrm{~cm}$ apart. The standard cultivation practices prescribed for wheat cultivation were adopted. The field observations were recorded in crop growing season. Experimental work on quality analysis were carried out at Wheat Quality Laboratory, Department of Food Science and Technology, MPKV, Rahuri

The mean performance revealed that the genotype viz., NIAW 2595, NIAW 2495, NIAW 2522, NIAW 301, NIAW 2539, NIAW 2587 and NIAW 2547 exhibited superior performance in all environments, indicating that these genotypes have more buffering capacity and suitable for early, timely and late sowing. The genotypes NIAW 2565, NIAW 2563, NIAW 34 and NIAW 2556 were found promising in E3 and E4 environments, hence, these genotypes can be considered in breeding programmes for improvement of wheat for late sowing.

Stability analysis revealed that, the mean sum of squares due to genotypes were significant for all the twelve characters across the environments, which indicated the presence of substantial variation in the material. The analysis also indicated significant variation among the environments for all the characters. The G x E interaction were significant for all twelve characters, which indicated that genotypes interacted differently with environmental variations for these characters (Table 1). Gowda et al. (2010) and Samrat et al. (2010) reported significant genotpe $\mathrm{x}$ environment interaction for grain yield, protein per cent, hectoliter weight and sedimentation value. Highly significant values of mean squares due to environments (linear) for all the characters indicated that the linear responses of genotypes to different environments. While mean square values due to $\mathrm{G} \times \mathrm{E}$ (pooled error) were also significant for 
all the characters. Karimizadeh et al. (2012) and Hasan et al. (2015) reported higher linear component for grain yield, while Khan et al. (2014) observed higher non linear component for this trait. It is evident from the present study that, genotypes expressed greater range of both bi and $\mathrm{S}^{2} \mathrm{di}$ for grain yield, which may be due to fixation of additive gene effects resulting from homozygosity and complementary.

For the characters viz., days to 50 per cent flowering, days to maturity and hectoliter weight, the E3 and E4 were better than E1 and E2 environments. However, environments E1 and E2 were better for tillers per plant, grain yield, earhead length, number of spikelets per earhead, number of grains earhead $^{-1}$, plant height and 1000-grain weight as well as for quality characters like protein content and sedimentation value.

The genotypes viz. NIAW 2565, NIAW 2556 and NIAW 2563 had average stability and are suitable for all four types of environments for grain yield. The NIAW 1994 had above average stability for grain yield and is well adapted to poor environment. The NIAW 2495, NIAW 2595, NIAW 2539 and NIAW 301 had below average stability for grain yield and is specifically adapted to favorable environment. These genotypes may be utilized in breeding programme for improvements under specific condition. These results are corroborated with the results of Bhoite et al. (2011) and Patil (2016)

From the present investigation, it was found that two genotypes NIAW 1994 and NIAW 2563 were showed stability for seven characters including grain yield. The genotypes NIAW 2565 and NIAW 2522 exhibited stability for six characters (Table 2). It is interesting to note here that, the genotype NIAW 1994 having higher mean performance and stability for grain yield, simultaneously also showed stability and high mean performance for number of spikelets earhead-1, earhead length, 1000-grain weight, number of tillers meter-1 $^{-1}$ and quality trait like hectoliter weight. Simillarly, the genotype NIAW 2563 showed high mean and average stability for grain yield, earhead length, number of tillers ${ }^{-1}$ meter and number of spikelets earhead-as well as protein content. While genotype NIAW 2565 exhibited average stability for grain yield, which also had below and above average stablity for number of spikelets/earhead, 1000-grain weight, earhead length along with quality parameters viz; protein content and hectoliter weight. Similar observations were also made by Hasan et al. (2015) and Patil (2016).

None of the genotypes was found to be ideal for wider adaptability for all the characters. The genotypes which shown stability for grain yield also showed simultaneous stability for one or more yield and quality components. Based on per se performance, regression coefficient and deviations from regression, the genotypes viz; NIAW 2539, NIAW 2556, NIAW 2563, NIAW 2565 and NIAW 1994 were found to be stable and adaptable to a wide range of environments, suggesting that these genotypes be better exploited in terms of grain yield and other important characters for improvement in wheat.

\section{REFERENCES:}

Bhoite, K.D., Rasal P.N. Gadekar D.A. and Gavhane V.N. (2011) Stabilty of grain yield in rainfed wheat. Ann. Agric. Res., 22 (1, 2): 1-3

Gowda, S. D. S., Singh G.P., Singh A.M., Deveshwar J.J. Ahalawat A. (2010) Stability analysis for physiological and quality 
parameters in wheat

(Triticumaestivum) Indian J. Agric. Sci.,80 (12): 1028-32.

Eberhart, S.A and Russell, W.A. (1966) Stability parameters for comparing varieties. Crop Sci., 6: 36-40.

Hasan, I., Rozalia, K., Moldovan, V and Ionut, R. (2015) Stability of grain yield and yield components in some winter wheat genotypes. Romanian j. Agric. Res., (32):1222-1227.

Karimizadeh, R., Mohammadi, M. Armion, M., Shefazadeh, M. K. and Chalajour, H. (2012) Determining heritability, reliability and stability of grain yield and yield-related components in durum wheat (Triticum durum L.). Bulg. J. Agric. Sci., 18: 595-607.

Khan, S., Khan. J., Khetran. M.A., Hanan. A., Kurd. A.A., Naseer. N.S and Jaffer. S (2014) Adaptability and stability of promising wheat genotypes for yield under rainfed conditions of highl and balochisthan. J. Animal and Plant Sci., 24(2): 521-525.

Patil, J. M. (2016) Effect of G X E interaction on morpho-physiological traits in bread wheat. A Ph.D. thesis submitted to MPKV, Rahuri. (Unpublished)

Samrat, D.S., Singh, G.P., Singh. A.M. Deveshwar, J.J. and Ahlawat, A. (2010) Stability analysis for physiological and quality parameters in wheat. Indian $J$. Agric. Sci., 80 (12):1028-32 
Table2. Stability parameters for yield, yield attributing and quality traits in bread wheat genotypes

\begin{tabular}{|c|c|c|c|c|c|c|c|c|c|c|c|c|c|}
\hline \multirow{2}{*}{ Sr.No } & \multirow{2}{*}{ Genotype } & \multicolumn{3}{|c|}{ Days to $50 \%$ flowering } & \multicolumn{3}{|c|}{ Days to maturity } & \multicolumn{3}{|c|}{ Plant height (cm) } & \multicolumn{3}{|c|}{ Number of tillers/meter } \\
\hline & & Mean & bi & $\mathbf{S}^{2} d i$ & Mean & bi & $\mathbf{S}^{2} d i$ & Mean & bi & $S^{2} d i$ & Mean & bi & $\mathbf{S}^{2} d i$ \\
\hline 1 & NIAW-2531 & 63.75 & 0.69 & $0.75^{*}$ & 105.83 & 0.87 & 0.40 & 72.41 & 1.01 & $53.9 * *$ & 44.58 & 0.65 & 5.16 \\
\hline 2 & NIAW-2595 & 65.66 & 0.93 & 0.57 & 111.91 & 1.28 & 0.29 & 86.06 & 0.85 & 0.46 & 54.16 & 1.35 & 0.98 \\
\hline 3 & NIAW-2495 & 66.08 & 1.16 & $2.06^{* *}$ & 113.08 & 1.18 & $0.73^{*}$ & 89.88 & 1.17 & 2.13 & 54.33 & 1.98 & $16.2^{*}$ \\
\hline 4 & NIAW-2565 & 65.58 & $0.24^{*}$ & -0.22 & 110.25 & $0.58^{*}$ & -0.10 & 87.51 & 1.06 & $22.2^{*}$ & 63.08 & $-0.63^{*}$ & -5.07 \\
\hline 5 & NIAW-2522 & 63.75 & 1.09 & -0.13 & 110.41 & 1.25 & $0.94^{*}$ & 85.48 & 0.80 & -5.13 & 78.80 & 1.36 & -0.08 \\
\hline 6 & NIAW-301 & 62.50 & 0.80 & $1.80^{* *}$ & 109.08 & $1.37^{*}$ & -0.13 & 76.78 & 1.21 & 1.96 & 71.76 & $2.27^{*}$ & -0.49 \\
\hline 7 & NIAW-2539 & 59.50 & 1.25 & $0.65^{*}$ & 105.75 & 1.24 & 0.35 & 75.37 & 1.20 & 3.49 & 69.43 & $1.90^{*}$ & -4.19 \\
\hline 8 & NIAW-2563 & 64.75 & 1.20 & 0.24 & 107.33 & 0.95 & -0.06 & 86.33 & 0.73 & -1.54 & 74.18 & $1.93^{*}$ & 3.07 \\
\hline 9 & NIAW-2556 & 64.58 & $0.55^{*}$ & -0.20 & 105.08 & 1.15 & -0.20 & 91.83 & 0.73 & $20.7^{*}$ & 64.06 & $-0.05^{*}$ & -3.95 \\
\hline 10 & NIAW-34 & 61.91 & $0.69^{*}$ & -0.24 & 103.00 & $0.21^{*}$ & -0.06 & 86.98 & 1.00 & $14.6^{*}$ & 82.20 & $0.05^{*}$ & -4.55 \\
\hline 11 & NIAW-2613 & 62.25 & 1.07 & $1.93^{* *}$ & 109.08 & 0.81 & 0.03 & 90.85 & 0.72 & -3.57 & 74.55 & $-0.32^{*}$ & 4.06 \\
\hline 12 & NIAW-1415 & 62.83 & 1.69 & $3.18^{* *}$ & 110.50 & 1.05 & 0.09 & 78.98 & 0.64 & 12.4 & 52.12 & $1.53^{*}$ & -3.03 \\
\hline 13 & NIAW-2463 & 62.50 & $0.57^{*}$ & -0.16 & 108.66 & 1.51 & $0.97^{*}$ & 90.46 & 1.37 & $30.8^{*}$ & 73.21 & $-0.01^{*}$ & -3.96 \\
\hline 14 & NIAW-1994 & 68.66 & $0.32^{*}$ & -0.29 & 106.00 & $0.11^{*}$ & -0.22 & 77.41 & $0.06^{*}$ & -2.61 & 90.00 & $0.20^{*}$ & -4.81 \\
\hline 15 & NIAW-2587 & 65.00 & $2.01^{*}$ & $1.74^{* *}$ & 113.70 & 1.68 & $0.89^{*}$ & 74.73 & 1.97 & 11.3 & 60.69 & $1.33^{*}$ & -4.28 \\
\hline 16 & NIAW-2547 & 63.83 & $1.74^{*}$ & 0.02 & 104.5 & $0.76^{*}$ & -0.22 & 82.60 & 1.48 & $28.7^{* *}$ & 58.05 & $2.45^{*}$ & 0.8 \\
\hline \multicolumn{2}{|c|}{ Population Mean } & 63.95 & & & 108.4 & & & 83.36 & & & 66.58 & & \\
\hline & SE \pm & 0.590 & & & 0.40 & & & 2.46 & & & 1.30 & & \\
\hline
\end{tabular}




\begin{tabular}{|c|c|c|c|c|c|c|c|c|c|c|c|c|c|}
\hline & & & & & \multicolumn{3}{|c|}{ spikelets/earhead } & \multicolumn{3}{|c|}{ grains/earhead } & \multirow[b]{2}{*}{ Mean } & \multirow[b]{2}{*}{$b i$} & \multirow[b]{2}{*}{$\mathbf{S}^{2} d i$} \\
\hline & & Mean & bi & $\mathbf{S}^{2} d i$ & Mean & bi & $\mathbf{S}^{2} d i$ & Mean & bi & $\mathbf{S}^{2} d i$ & & & \\
\hline 1 & NIAW-2531 & 10.13 & 2.06 & $0.35^{* *}$ & 12.45 & 1.17 & -0.11 & 51.98 & 1.91 & $9.18^{* *}$ & 598.91 & 1.30 & -1418.0 \\
\hline 2 & NIAW-2595 & 8.225 & 1.81 & -0.07 & 13.99 & $1.39 *$ & -0.20 & 50.11 & 1.97 & 0.07 & 791.00 & 2.27 & 931.70 \\
\hline 3 & NIAW-2495 & 10.43 & 1.17 & -0.07 & 15.66 & 1.53 & -0.08 & 47.98 & 1.03 & $6.2^{*}$ & 899.08 & $3.16^{*}$ & 655.80 \\
\hline 4 & NIAW-2565 & 10.10 & $3.01^{*}$ & -0.07 & 15.25 & 1.50 & -0.04 & 49.30 & 2.27 & $8.05^{* *}$ & 826.25 & $-1.03^{*}$ & -756.60 \\
\hline 5 & NIAW-2522 & 11.06 & 1.25 & -0.09 & 17.00 & 2.07 & 0.37 & 52.23 & $2.66^{*}$ & -1.30 & 757.50 & 3.01 & 3289.0 \\
\hline 6 & NIAW-301 & 10.04 & 0.92 & -0.06 & 15.80 & 0.51 & 0.35 & 50.18 & 1.88 & $5.51^{*}$ & 821.91 & $3.54^{*}$ & -824.30 \\
\hline 7 & NIAW-2539 & 9.125 & -1.58 & -0.02 & 11.99 & 2.29 & 0.17 & 45.42 & 1.26 & 0.23 & 782.08 & 1.83 & -1122.0 \\
\hline 8 & NIAW-2563 & 10.60 & 0.42 & 0.06 & 16.24 & $-0.49 *$ & -0.18 & 50.87 & $-0.57^{*}$ & 2.06 & 792.08 & -1.09 & 2664.05 \\
\hline 9 & NIAW-2556 & 9.817 & 1.37 & 0.03 & 16.14 & $0.09 *$ & -0.19 & 50.26 & 1.53 & $4.38^{*}$ & 777.83 & -0.94 & $8495.3^{* *}$ \\
\hline 10 & NIAW-34 & 9.167 & 1.15 & -0.05 & 14.70 & -0.09 & $0.42^{*}$ & 53.50 & 0.48 & 1.49 & 760.33 & $-2.11^{*}$ & 636.26 \\
\hline 11 & NIAW-2613 & 10.40 & $-0.03^{*}$ & -0.09 & 13.88 & -2.18 & $1.04 * *$ & 54.00 & -0.29 & $6.88^{* *}$ & 650.25 & $-1.63^{*}$ & -825.86 \\
\hline 12 & NIAW-1415 & 10.23 & $-0.25^{*}$ & -0.09 & 17.96 & 2.02 & $0.57^{*}$ & 61.41 & 1.14 & $21.11^{* *}$ & 699.16 & $0.14^{*}$ & -1492.9 \\
\hline 13 & NIAW-2463 & 9.542 & -0.17 & -0.07 & 14.64 & 0.33 & $0.69 *$ & 57.51 & -1.57 & $8.79^{* *}$ & 661.83 & -0.70 & 2518.65 \\
\hline 14 & NIAW-1994 & 10.11 & 0.65 & -0.09 & 16.43 & 1.18 & -0.04 & 51.48 & $-0.09 *$ & -1.44 & 1090.0 & 0.60 & -1395.0 \\
\hline 15 & NIAW-2587 & 11.03 & 2.06 & 0.02 & 15.87 & $3.59^{*}$ & -0.19 & 49.38 & 0.87 & 0.71 & 744.75 & 3.75 & 4907.90* \\
\hline 16 & NIAW-2547 & 10.15 & 2.14 & -0.05 & 15.59 & 1.08 & -0.08 & 50.88 & 1.53 & $5.14^{*}$ & 747.00 & 3.91 & $4612.30^{*}$ \\
\hline \multicolumn{2}{|c|}{ Population Mean } & 10.01 & & & 15.23 & & & 51.66 & & & 775.0 & & \\
\hline & SE \pm & 0.152 & & & 0.345 & & & 1.450 & & & 31.40 & & \\
\hline
\end{tabular}

Cont... 


\begin{tabular}{|c|c|c|c|c|c|c|c|c|c|c|c|c|c|}
\hline \multirow[t]{2}{*}{ Sr.No } & \multirow[t]{2}{*}{ Genotype } & \multicolumn{3}{|c|}{1000 grain weight (g) } & \multicolumn{3}{|c|}{ Protein content $(\%)$} & \multicolumn{3}{|c|}{$\begin{array}{c}\text { Hectolitre weight } \\
(\mathrm{kg} / \mathrm{hl})\end{array}$} & \multicolumn{3}{|c|}{$\begin{array}{l}\text { Sedimentation value } \\
(\mathrm{ml})\end{array}$} \\
\hline & & Mean & bi & $\mathbf{S}^{2} \mathbf{d i}$ & Mean & bi & $\mathbf{S}^{2} d i$ & Mean & $B i$ & $\mathbf{S}^{2} d i$ & Mean & $B i$ & $\mathbf{S}^{2} d i$ \\
\hline 1 & NIAW-2531 & 41.66 & 3.13 & $3.35^{* *}$ & 11.15 & 1.29 & 0.11 & 77.79 & 0.94 & 0.09 & 38.83 & 0.75 & $1.46^{*}$ \\
\hline 2 & NIAW-2595 & 39.75 & $6.42^{*}$ & -0.32 & 12.17 & 2.44 & -0.07 & 78.08 & 0.80 & 0.17 & 46.00 & 1.57 & -0.17 \\
\hline 3 & NIAW-2495 & 42.83 & $4.69^{*}$ & -0.64 & 11.29 & -0.09 & 0.26 & 77.68 & 0.94 & $1.60^{* *}$ & 39.50 & 0.52 & $8.52^{* *}$ \\
\hline 4 & NIAW-2565 & 41.16 & $-1.42^{*}$ & 0.13 & 12.58 & $3.59^{*}$ & -0.07 & 79.14 & 1.07 & -0.02 & 46.08 & 2.58 & 2.35 \\
\hline 5 & NIAW-2522 & 40.00 & 2.04 & 0.06 & 11.3 & -0.51 & -0.07 & 78.64 & 0.21 & $0.55^{*}$ & 40.50 & 0.40 & $4.54 * *$ \\
\hline 6 & NIAW-301 & 40.50 & 2.05 & -0.18 & 11.61 & -0.09 & -0.12 & 78.50 & 0.90 & 0.11 & 41.25 & -0.24 & $6.69^{* *}$ \\
\hline 7 & NIAW-2539 & 39.75 & $3.87^{*}$ & -0.46 & 11.15 & 0.74 & -0.13 & 77.12 & 0.89 & -0.11 & 38.91 & 1.04 & 0.40 \\
\hline 8 & NIAW-2563 & 39.58 & $-2.59^{*}$ & 0.17 & 12.51 & 0.55 & 0.12 & 76.18 & 0.92 & $2.50^{* *}$ & 45.9 & 1.45 & $5.35^{* *}$ \\
\hline 9 & NIAW-2556 & 40.25 & $-3.97^{*}$ & 0.36 & 13.13 & 1.61 & 0.02 & 77.75 & $0.46^{*}$ & -0.12 & 50.58 & 1.54 & 1.48 \\
\hline 10 & NIAW-34 & 40.00 & -0.88 & 0.06 & 12.51 & $2.48^{*}$ & -0.12 & 76.64 & 0.96 & $0.49^{*}$ & 46.08 & 1.35 & $6.93^{* *}$ \\
\hline 11 & NIAW-2613 & 40.00 & $-2.57^{*}$ & 0.14 & 12.92 & 1.42 & 0.25 & 78.71 & 1.02 & $0.61^{*}$ & 51.75 & 1.39 & $30.6^{* *}$ \\
\hline 12 & NIAW-1415 & 39.75 & 0.04 & -0.52 & 11.48 & 1.17 & -0.07 & 76.43 & $1.47^{*}$ & -0.11 & 40.25 & 1.20 & $7.04^{* *}$ \\
\hline 13 & NIAW-2463 & 40.33 & $-1.96^{*}$ & -0.48 & 11.15 & 0.46 & -0.02 & 78.78 & 1.21 & 0.03 & 38.58 & 0.56 & $2.00^{*}$ \\
\hline 14 & NIAW-1994 & 44.08 & 0.68 & 0.13 & 11.63 & -0.78 & -0.04 & 78.66 & 0.96 & -0.08 & 42.75 & $-0.24^{*}$ & 0.11 \\
\hline 15 & NIAW-2587 & 40.75 & 5.86 & $3.95^{* *}$ & 11.29 & 0.83 & -0.11 & 76.81 & 1.67 & $2.80^{*}$ & 42.16 & 1.64 & $8.00 * *$ \\
\hline 16 & NIAW-2547 & 40.25 & \multirow[t]{3}{*}{0.61} & -0.52 & 12.11 & \multirow[t]{3}{*}{0.88} & $0.39 *$ & 76.67 & \multirow{3}{*}{\multicolumn{2}{|c|}{\begin{tabular}{l|l}
$1.59^{*}$ & -0.16 \\
\end{tabular}}} & 44.41 & 0.51 & $11.24^{* *}$ \\
\hline \multicolumn{2}{|c|}{ Population Mean } & 40.67 & & & 11.88 & & & 77.73 & & & 43.35 & \multirow{2}{*}{0.01} & \\
\hline & SE \pm & 0.576 & & & 0.234 & & & 0.482 & & & 1.500 & & \\
\hline
\end{tabular}

$(*, * *$ significant at $5 \%$ and $1 \%$ level respectively) 
Table 1.Pooled analysis of variance of for yield, yield attributing and quality traits in wheat genotypes

\begin{tabular}{|c|c|c|c|c|c|c|c|}
\hline \multirow{2}{*}{$\begin{array}{l}\text { Source of } \\
\text { variation }\end{array}$} & \multirow{2}{*}{$\begin{array}{l}\text { Degrees } \\
\text { of } \\
\text { freedom }\end{array}$} & \multicolumn{6}{|c|}{ Mean Squares } \\
\hline & & $\begin{array}{c}\text { Days to } 50 \\
\text { percent } \\
\text { flowering }\end{array}$ & $\begin{array}{l}\text { Days to } \\
\text { maturity }\end{array}$ & $\begin{array}{c}\text { Plant } \\
\text { height } \\
\text { (cm) }\end{array}$ & $\begin{array}{c}\text { Number of } \\
\text { tillers/meter }\end{array}$ & $\begin{array}{c}\text { Earhead } \\
\text { length } \\
\text { (cm) }\end{array}$ & $\begin{array}{c}\text { Number of } \\
\text { spikelets/earhead }\end{array}$ \\
\hline Repl. within Env. & 8 & 0.244 & 0.262 & 7.929 & 7.138 & 0.083 & 0.259 \\
\hline Genotypes & 15 & $17.88^{* *}$ & $37.85^{* *}$ & $167.5^{* *}$ & $604.0 * *$ & $2.105^{\star *}$ & $9.913 * *$ \\
\hline Environments & 3 & $234.0 * *$ & $190.0^{* *}$ & $1366^{* *}$ & $1919^{* *}$ & $1.106^{* *}$ & $6.978 * *$ \\
\hline Geno. xEnv. & 45 & $4.497^{* * \# \#}$ & $2.621^{\text {**\#\# }}$ & $28.52^{\# \#}$ & 124.9 **\#\# & 0.139 *\# & $1.019^{* * \# \#}$ \\
\hline Env.+ (geno.xenv) & 48 & $18.84^{* *}$ & $14.33^{* *}$ & $112.1^{* *}$ & $237.1^{* *}$ & $0.200 * *$ & $1.392 * *$ \\
\hline Env. (linear) & 1 & $702.1^{* *}$ & $570.0^{* *}$ & $4098^{* *}$ & $5758^{* *}$ & $3.319^{* *}$ & $20.93^{* *}$ \\
\hline Geno.xEnv.(linear) & 15 & $11.26^{* *}$ & $6.694^{* *}$ & $46.73^{*}$ & $364.1^{* *}$ & $0.270^{* *}$ & $2.297^{* *}$ \\
\hline Pooled Deviation & 32 & $1.045^{\# \#}$ & $0.548^{\#}$ & $18.20^{\# \#}$ & 5.034 & 0.069 & 0.357 \# \\
\hline Pooled Error & 120 & 0.318 & 0.321 & 6.209 & 5.164 & 0.092 & 0.196 \\
\hline
\end{tabular}


Table contd.....

\begin{tabular}{|c|c|c|c|c|c|c|c|}
\hline \multirow{2}{*}{$\begin{array}{l}\text { Source of } \\
\text { variation }\end{array}$} & \multirow{2}{*}{$\begin{array}{l}\text { Degrees } \\
\text { of } \\
\text { freedom }\end{array}$} & \multicolumn{6}{|c|}{ Mean Squares } \\
\hline & & $\begin{array}{c}\text { Number of } \\
\text { grains/Earhead }\end{array}$ & $\begin{array}{c}\text { Grain } \\
\text { yield/plot } \\
\text { (g) }\end{array}$ & $\begin{array}{l}1000 \text { grain } \\
\text { weight (g) }\end{array}$ & $\begin{array}{l}\text { Protein } \\
\quad \%\end{array}$ & $\begin{array}{c}\text { Hectolitre } \\
\text { weight (kg/hl) }\end{array}$ & $\begin{array}{l}\text { Sedimentation } \\
\text { value (ml) }\end{array}$ \\
\hline Repl. within Env. & 8 & 0.873 & 2092 & 0.246 & 0.237 & 0.480 & 1.218 \\
\hline Genotypes & 15 & $55.92^{* *}$ & $4997^{* *}$ & $6.170^{* *}$ & $1.847^{* *}$ & $3.766^{* *}$ & $67.75^{* *}$ \\
\hline Environments & 3 & $144.3^{* *}$ & $5259 * *$ & $16.06^{* *}$ & $1.243^{* *}$ & $47.25^{* *}$ & $138.5^{* *}$ \\
\hline Geno. x Env & 45 & $16.37^{* * \# \#}$ & $1661^{* * \# \#}$ & $10.83^{* * \# \#}$ & $0.221^{* \#}$ & $0.900 * \# \#$ & $9.537^{* \# \#}$ \\
\hline $\begin{array}{c}\text { Env.+ (geno. } x \\
\text { env) }\end{array}$ & 48 & $24.37^{* *}$ & $1886^{* *}$ & $11.16^{* *}$ & 0.285 & $3.797^{* *}$ & $17.60^{* *}$ \\
\hline Env. (linear) & 1 & $433.1^{* *}$ & $1577^{* *}$ & $48.20^{* *}$ & $3.729 * *$ & $141.7^{* *}$ & $415.7^{* *}$ \\
\hline Geno.xEnv.(linear) & 15 & $35.66^{* *}$ & $4352^{* *}$ & $30.38^{* *}$ & 0.311 & $1.209^{*}$ & $14.21^{*}$ \\
\hline Pooled Deviation & 32 & $6.315^{\# \#}$ & $2962^{\#}$ & 0.996 & 0.165 & $0.698^{\# \#}$ & $6.748^{\# \#}$ \\
\hline Pooled Error & 120 & 1.540 & 1628 & 0.698 & 0.138 & 0.157 & 0.674 \\
\hline
\end{tabular}

${ }^{*},{ }^{* *}$ significant at $5 \%$ and $1 \%$ level respectively when tested against pooled deviation; while, \#, \#\# significant at $5 \%$ and $1 \%$ level respectivelywhen tested against pooled error) 